\section{Cellular control}

\author{
Sydney Shall
}

ADP-Ribosylation of Proteins: Enzymology and Biological Significance. By Felix R. Althaus and Christoph Richter. Springer-Verlag: 1987. Pp.237. DM198.

Control of cellular reactions is often realized by the covalent modification of previously synthesized enzymes, receptors, structural proteins and so on. The covalent ADP-ribosylation of proteins is one such regulatory mechanism.

ADP-ribosylation may be either monomeric or polymeric. Mono-ADP-ribosyl transferases include an array of bacterial toxins and a number of endogenous cellular enzymes. The bacterial toxins are the easiest to study - we know more about them than about the cell enzymes - and are described in the first part of this book. Diphtheria, Pseudomonas, cholera, pertussis (whooping cough), E. coli and some minor botulinum toxins all consist in part of ADP-ribosyl transferase enzymes. These toxins (enzymes) transfer ADPribose from NAD to a very specific amino acid residue in a very specific target protein - all of which are apparently so-called G-proteins or GTP-binding proteins. Because endogenous enzymes with similar specificities have been described it is likely that ADP-ribosylation will emerge as an important mode of regulation of many $\mathrm{G}$-proteins.

The other part of the book deals with poly-ADP-ribosylation reactions on chromatin proteins. Felix Althaus has a strong prejudice in favour of the notion that poly-ADP-ribosylation reactions modulate chromatin conformation. He has argued this idea frequently and with great conviction, and does so again here. However, although I found the exercise of preferences and prejudices most admirable and stimulating, and some elegant experiments with purified components support the notion, the book describes no convincing observations which show that poly-ADP-ribosylation reactions modify chromatin structure in intact cells. Of course, poly-(ADP-ribose) is a large polyanion, and this implies that such a polymer is very likely to modify chromatin structure. In my view, this last argument is at present the best one in support of Althaus's views.

The authors also discuss the demonstration, some years ago, that poly-ADPribosylation reactions are an integral part of the cellular response to DNA-damaging agents, probably in direct proportion to the steady-state level of DNA nicks and breaks, and probably also only in a regulatory not in a mechanistic role. The practical therapeutic potential of these observations is currently being explored in a number of laboratories, but the precise molecular explanation of the function of poly-(ADP-ribose) in DNA repair is still being defined. My colleagues and I have published some evidence indicating that perhaps poly-(ADP-ribose) polymerase (indirectly?) modulates the activity of DNA ligase 11. For the most part, this history is accurately and clearly described in the book. However, it seems to me that the authors have not discriminated sufficiently clearly between data derived from intact cells (or organisms) and those derived from isolated or purified components. Although both types of data are valid, they are not at all equivalent.

A comprehensive monograph takes time to produce and the field has not waited upon the publishers; the flowers

have continued to bloom. Thus, the molecular biology of poly-(ADP-ribose) polymerase is not recorded in this book; the gene has been cloned in at least six laboratories during 1987. Also, the work on botulinum toxins $C$ and $D$ is not described. These omissions merely indicate the rapid pace of advance; the number of publications in this field has been growing exponentially since 1980 .

Althaus and Richter have produced a well-written, comprehensive and stimulating review of this rapidly expanding area of research. Although it must be read critically, the book will be the standard account of ADP-ribosylation of proteins for many years to come.

Sydney Shall is a Professor in the School of Biological Sciences, University of Sussex, Brighton BN1 9QG, UK.

\section{Never spare the rod}

\section{David W. Kingsbury}

The Rhabdoviruses. Edited by Robert R. Wagner. Plenum: 1987. Pp.544. $\$ 89.50$ (North America), $\$ 107.40$ (elsewhere)

FOR biological mystery and glamour, consider the rhabdoviruses. This large family of ultramicroscopic rodlets (rhabdos, rod) has evidently been a companion and occasional pathogen of multicellular organisms for eons, considering the impressive variety of phyla afflicted by its members. A long evolutionary history is indicated by the existence of rhabdoviruses that infect plants (including food staples), insects (such as the hereditary sigma virus of Drosophila), cold-blooded vertebrates (salmon and trout among them) and warm-blooded vertebrates (vesicular stomatitis virus of cattle and swine has a significant impact on human affairs).

The most serious human pathogen among the rhabdoviruses is the causative agent of rabies. Louis Pasteur tamed la rage by selecting avirulent rabies virus mutants and injecting them into infected subjects. Luckily for him, for those injectees and for the rest of us, the long incubation period of the disease opens a sufficiently wide window of time for the mutant viruses to stimulate curative antibodies. Pasteur's achievement was a cornerstone of the science of immunology, and it established a precedent for the development of attenuated vaccines prophylactic against many other viruses and bacteria.

Rhabdoviruses seem simple genetically, possessing only five or six genes in a single strand of RNA. Adding complexity, the RNA strand is complementary to the messenger RNAs that specify viral proteins, placing the rhabdoviruses in the broad category of negative-stranded viruses. This modus vivendi entails the dedication of precious genetic information to specifying enzymatic proteins that can transcribe and replicate the viral genome. But transcription offers a versatile means of regulating gene expression, a benefit that has evidently been worth the price, given the evolutionary success of negative-strand viruses. Despite much progress, many important questions about their replication are unanswered, because they do not engage in genetic recombination, nor are they easily manipulated biochemically or by recombinant DNA technology.

The Rhabdoviruses is the latest in a series, The Viruses, comprising volumes of reviews devoted to individual virus families, edited by $\mathrm{H}$. Fraenkel-Conrat and R.R. Wagner. This volume maintains the high standards of the series, which updates and expands upon an earlier set, Comprehensive Virology, a product of the same editorial team.

Wagner has assembled 11 separately authored reviews that assess progress in the field, giving due weight to controversies and unsolved problems. The editor himself contributes two overview chapters. He and his coauthors are all wellknown authorities, actively engaged in research on their topics, and are skilful communicators. Most of the chapters deal with the molecular genetics of the popular experimental model of the family, vesicular stomatitis virus (VSV), but there is a chapter on rabies virus pathogenesis, one on plant rhabdoviruses, and a brief review of the ecology of rhabdoviruses that infect vertebrates. In addition, the chapters on VSV are enriched and balanced by abundant references to related work on other members of the family, making the book valuable not only to specialists but also to a broader audience.

David W. Kingsbury is in the Department of Virology and Molecular Biology, St Jude Children's Research Hospital, PO Box 318, Memphis, Tennessee 38101, USA. 